\title{
Control of Nonlinear Bilateral Teleoperation Systems Subject to Disturbances
}

\author{
Alireza Mohammadi, Mahdi Tavakoli and Horacio J. Marquez
}

\begin{abstract}
Teleoperation systems, consisting of a pair of master and slave robots are subject to different types of disturbances such as joint frictions, varying contact points, unmodeled dynamics and unknown payloads. Such disturbances, when unaccounted for, cause poor teleoperation transparency and even instability. This paper presents a novel nonlinear bilateral control scheme, based on the concept of disturbance observer based control, to counter these disturbances and their negative effects on the teleoperation systems. The proposed disturbance observer based bilateral control law is able to acheive global asymptotic force tracking, and global exponential position and disturbance tracking in the presence of various disturbances. The minimum exponential convergence rate of the position and the disturbance tracking errors can be tuned by the controller parameters. Simulations are presented to show the effectiveness of the proposed control scheme.
\end{abstract}

\section{INTRODUCTION}

Every teleoperation system consists of a master robot and a slave robot. The master interacts with a human operator and the slave interacts with a remote environment. If force feedback from the slave side to the master side is provided, the system is called a bilateral teleoperation system to distinguish it from a unilateral teleoperation system. A bilateral teleoperation system is said to be transparent if the slave robot precisely follows the position of the master robot and the master robot faithfully displays the slave-environment contact force to the human operator.

The most successful control scheme in achieving a fully transparent teleoperation system is the 4-channel architecture [1], [2], which is mostly suitable for teleoperation systems with fixed linear models. Physical robots, however, are nonlinear systems subject to various disturbances, such as unknown dynamics, joint frictions, unknown payloads, etc. [3]. Such disturbances, when unaccounted for, cause poor teleoperation transparency and even instability. One way to suppress these disturbances, is to employ disturbance observers [4]. Disturbance observer based control has been used in applications such as independent robot joint control [5], and robot joint friction estimation and compensation [6], [7].

A considerable part of the existing literature on disturbance observer design for robotic applications uses linearized models or linear system techniques [8], [9]. In order to overcome

This work was supported by the Natural Sciences and Engineering Research Council of Canada (NSERC)

A. Mohammadi was with the Department of Electrical \& Computer Engineering, University of Alberta, Edmonton, AB T6G 2V4 Canada. He is now with the Edward S. Rogers Sr. Department of Electrical \& Computer Engineering, University of Toronto, Toronto, ON M5S 3G4 Canada. Email: alireza.mohammadi@mail.utoronto.ca

M. Tavakoli is with the Department of Electrical \& Computer Engineering, University of Alberta, Edmonton, AB T6G 2V4 Canada. Email: tavakoli@ece.ualberta.ca

H. J. Marquez is with the Department of Electrical \& Computer Engineering, University of Alberta, Edmonton, AB T6G 2V4 Canada. Email: marquez@ece.ualberta.ca the linear disturbance observer limitations for the highly nonlinear and coupled dynamics of robotic manipulators, Chen et al. proposed a nonlinear disturbance observer for nonlinear robotic manipulators and designed it such that no acceleration measurement was needed [10]. However, the closed-loop stability of the overall system including the disturbance observer and the controller was not investigated. The investigation of the stability and performance of a master-slave teleoperation system under disturbance observer based control is even more challenging and not studied either. While a distrubance observer based controller was designed for bilateral teleoperation systems in [11], the master and the slave robots were considered to be linear with only one DOF. This serves as the motivation to look for a disturbance observer based control law for nonlinear and $n$-DOF teleoperation systems.

This paper ${ }^{1}$ addresses the problem of disturbance observer based control of nonlinear teleoperation systems. A disturbance observer based control law will be proposed and incorporated into the framework of the 4-channel teleoperation architecture. Under the proposed control law, full transparency and exponential disturbance and position tracking are achieved.

The organization of this paper is as follows. Section II introduces the nonlinear model of the teleoperation systems and 4-channel bilateral control architecture. Section III proposes a novel disturbance observer based controller for nonlinear teleoperation systems subject to various disturbances. The teleoperation system transparency is also addressed in this section. Finally, simulations in section IV show the efficiency of the proposed control scheme as compared with the case where no disturbance observer is employed.

\section{NONLINEAR MODEL OF A TELEOPERATION SYSTEM}

The dynamical models investigated in this paper and our proposed control law in section III will be in the Cartesian space. This enables us to make a teleoperation system transparent without requiring the master and the slave robots to have similar kinematics and dynamics.

\section{A. Model of a teleoperation system in the Cartesian space}

The dynamic equations describing the motions of the endeffectors of $n$-DOF master and slave robots, which interact with the human operator and the remote environment, in the presence of disturbances can be written as

$$
\begin{aligned}
\mathbf{M}_{x m}\left(\mathbf{q}_{m}\right) \ddot{\mathbf{x}}_{m}+\mathbf{N}_{x m}\left(\mathbf{q}_{m}, \dot{\mathbf{q}}_{m}\right) & =\mathbf{f}_{m}+\mathbf{f}_{h}+\mathbf{d}_{m} \\
\mathbf{M}_{x s}\left(\mathbf{q}_{s}\right) \ddot{\mathbf{x}}_{s}+\mathbf{N}_{x s}\left(\mathbf{q}_{s}, \dot{\mathbf{q}}_{s}\right) & =\mathbf{f}_{s}-\mathbf{f}_{e}+\mathbf{d}_{s}
\end{aligned}
$$

\footnotetext{
${ }^{1} \mathrm{~A}$ more complete version of this paper can be found in [12].
} 
where $\mathbf{q}_{m}, \mathbf{q}_{s}, \mathbf{x}_{m}, \mathbf{x}_{s}, \mathbf{M}_{x m}\left(\mathbf{q}_{m}\right), \quad \mathbf{M}_{x s}\left(\mathbf{q}_{s}\right), \quad \mathbf{N}_{x m}\left(\mathbf{q}_{m}, \dot{\mathbf{q}}_{m}\right)$ and $\mathbf{N}_{x s}\left(\mathbf{q}_{s}, \dot{\mathbf{q}}_{s}\right)$ are the vectors of joint positions, the position/orientation (pose) vectors of the end-effectors in the Cartesian space, the inertia matrices in the Cartesian space, and the vectors of Coriolis/centrifugal and gravity forces in the Cartesian space. The subscripts $m$ and $s$ refer to the master and the slave robots, respectively. Also, $\mathbf{f}_{m}, \mathbf{f}_{s} \in R^{6 \times 1}$ are the control forces applied to the master and the slave end-effectors in the Cartesian space, $\mathbf{f}_{h}, \mathbf{f}_{e} \in R^{6 \times 1}$ are the forces exerted to the master and the slave end-effectors by the human operator and the remote environment, and $\mathbf{d}_{m}, \mathbf{d}_{s} \in$ $R^{6 \times 1}$ are the disturbance forces exerted to the master and the slave end-effectors in the Cartesian space. These disturbance forces represent the lumped effect of all disturbances acting on the master and the slave robots such as joint frictions, varying contact points, unmodeled dynamics and unknown payloads in the Cartesian space.

\section{B. 4-channel control architecture}

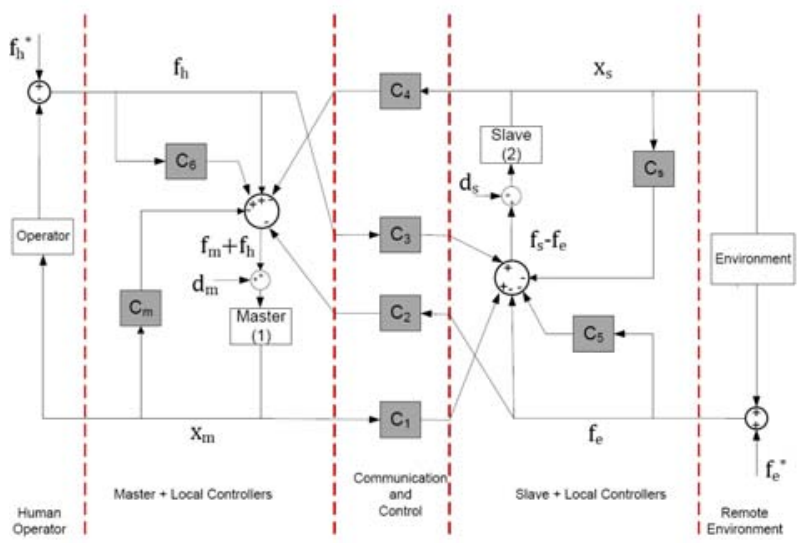

Fig. 1. 4-channel teleoperation control architecture.

The 4-channel control architecture is shown in Figure 1. Exogenous signals $\mathbf{f}_{h}^{*}$ and $\mathbf{f}_{e}^{*}$ are exerted by the human operator and the remote environment, respectively. The signals $\mathbf{x}_{m}$, $\mathbf{x}_{s}, \mathbf{f}_{h}, \mathbf{f}_{e}, \mathbf{f}_{m}, \mathbf{f}_{s}, \mathbf{d}_{m}$ and $\mathbf{d}_{s}$ are as defined in (1)-(2). Position information is exchanged between the master and the slave via the position channels $\mathbf{C}_{1}$ and $\mathbf{C}_{4}$. Force information is exchanged through the force channels $\mathbf{C}_{2}$ and $\mathbf{C}_{3}$. In addition, $\mathbf{C}_{m}$ and $\mathbf{C}_{s}$ are local master and slave (position) controllers. Lastly, $\mathbf{C}_{5}$ and $\mathbf{C}_{6}$ provide the master and the slave with local force feedback from the human operator and the remote environment, respectively. In the conventional design of 4channel controllers, it is assumed that $\mathbf{d}_{m}=\mathbf{0}$ and $\mathbf{d}_{s}=\mathbf{0}$. We are, however, going to deal with the case where these disturbances are not zero.

In a fully transparent teleoperation system we have $\mathbf{x}_{s}=\mathbf{x}_{m}$ and $\mathbf{f}_{h}=\mathbf{f}_{e}$. If the master and the slave are represented by LTI impedances $\mathbf{Z}_{m}(s)$ and $\mathbf{Z}_{s}(s)$, the 4-channel teleoperation system of Figure 1 becomes fully transparent in the absence of disturbances and delays if the controllers are chosen as [13]

$$
\begin{aligned}
& \mathbf{C}_{1}=\mathbf{Z}_{s}+\mathbf{C}_{s} \\
& \mathbf{C}_{2}=\mathbf{I}+\mathbf{C}_{6} \\
& \mathbf{C}_{3}=\mathbf{I}+\mathbf{C}_{5} \\
& \mathbf{C}_{4}=-\mathbf{Z}_{m}-\mathbf{C}_{m}
\end{aligned}
$$

\section{DISTURBANCE OBSERVER BASED CONTROL OF TELEOPERATION SYSTEMS}

In this section, first the concept of disturbance observer based control is introduced. Next, control laws are developed based on this concept and incorporated into the 4-channel architecture for a nonlinear teleoperation system.

\section{A. Disturbance observer based control concept}

Figure 2 depicts a disturbance observer which is used for attenuating disturbances acting on a single robot. The vector d represents the lumped disturbance, which deteriorates the tracking performance of the robot control system. The disturbance observer role is to estimate this disturbance as closely as possible. The estimated disturbance $\hat{\mathbf{d}}$ is then subtracted from the control signal $\mathbf{f}$ to cancel out or minimize the effect of the disturbance.

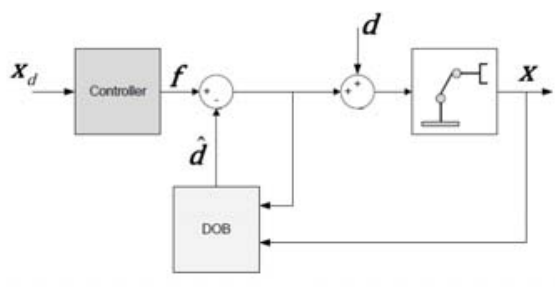

Fig. 2. Disturbance observer used for attenuating disturbances acting on a single robot.

\section{B. Proposed teleoperation control laws}

Extending the idea of disturbance observer based control of a single robot to a master-slave teleoperation system, we will design a disturbance observer for each of the master and the slave robots in order to estimate and cancel out the disturbances.

Assume that the master and the slave disturbances are estimated to be $\hat{\mathbf{d}}_{m}$ and $\hat{\mathbf{d}}_{s}$, respectively. We propose the following nonlinear control laws for the master and slave robots described by (1) and (2), respectively:

$$
\begin{aligned}
\mathbf{f}_{m}= & \mathbf{M}_{x m}\left(\mathbf{q}_{m}\right)\left[-\mathbf{C}_{m} \mathbf{x}_{m}-\mathbf{C}_{2} \mathbf{f}_{e}-\mathbf{C}_{4} \mathbf{x}_{s}+\mathbf{C}_{6} \mathbf{f}_{h}+\mathbf{f}_{h}\right] \\
& +\mathbf{N}_{x m}\left(\mathbf{q}_{m}, \dot{\mathbf{q}}_{m}\right)-\mathbf{f}_{h}-\hat{\mathbf{d}}_{m} \\
\mathbf{f}_{s}= & \mathbf{M}_{x s}\left(\mathbf{q}_{s}\right)\left[-\mathbf{C}_{s} \mathbf{x}_{s}+\mathbf{C}_{1} \mathbf{x}_{m}+\mathbf{C}_{3} \mathbf{f}_{h}-\mathbf{C}_{5} \mathbf{f}_{e}-\mathbf{f}_{e}\right] \\
& +\mathbf{N}_{x s}\left(\mathbf{q}_{s}, \dot{\mathbf{q}}_{s}\right)+\mathbf{f}_{e}-\hat{\mathbf{d}}_{s}
\end{aligned}
$$

where $\mathbf{C}_{m}, \mathbf{C}_{s}, \mathbf{C}_{1}, \ldots$, and $\mathbf{C}_{6}$ are some LTI controllers used in the above nonlinear control laws. Note the use of disturbance estimates $\hat{\mathbf{d}}_{m}$ and $\hat{\mathbf{d}}_{s}$ in the proposed control laws.

The disturbance observer based control laws (4) and (5), when applied to the master and slave described by (1)-(2), result in the following closed-loop equations for the two robots: 


$$
\begin{aligned}
\ddot{\mathbf{x}}_{m}= & -\mathbf{C}_{m} \mathbf{x}_{m}-\mathbf{C}_{2} \mathbf{f}_{e}-\mathbf{C}_{4} \mathbf{x}_{s}+\mathbf{C}_{6} \mathbf{f}_{h} \\
& +\mathbf{f}_{h}+\mathbf{M}_{x m}^{-1}\left(\mathbf{q}_{m}\right) \Delta \mathbf{d}_{m} \\
\ddot{\mathbf{x}}_{s}= & -\mathbf{C}_{s} \mathbf{x}_{s}+\mathbf{C}_{1} \mathbf{x}_{m}+\mathbf{C}_{3} \mathbf{f}_{h}-\mathbf{C}_{5} \mathbf{f}_{e} \\
& -\mathbf{f}_{e}+\mathbf{M}_{x s}^{-1}\left(\mathbf{q}_{s}\right) \Delta \mathbf{d}_{s}
\end{aligned}
$$

where $\Delta \mathbf{d}_{m}=\mathbf{d}_{m}-\hat{\mathbf{d}}_{m}$ and $\Delta \mathbf{d}_{s}=\mathbf{d}_{s}-\hat{\mathbf{d}}_{s}$ are the master and the slave disturbance estimation errors, respectively.

Remark 1. When $\Delta \mathbf{d}_{m}=\mathbf{0}$ and $\Delta \mathbf{d}_{s}=\mathbf{0}$, i.e., under ideal disturbance tracking, the closed-loop system equations (6) and (7) describe an $n$-DOF teleoperation system without disturbances, similar to the one shown in Figure 1 with the master and slave robots represented by identity inertia matrices, i.e., $\mathbf{Z}_{m}(s)=s^{2} \mathbf{I}$ and $\mathbf{Z}_{s}(s)=s^{2} \mathbf{I}$. $\diamond$

Let us choose the master and the slave local position controllers in (4) and (5) to be of proportional-derivative type:

$$
\begin{aligned}
\mathbf{C}_{m} & =\mathbf{K}_{m v} s+\mathbf{K}_{m p} \\
\mathbf{C}_{s} & =\mathbf{K}_{s v} s+\mathbf{K}_{s p}
\end{aligned}
$$

where $\mathbf{K}_{m v}, \mathbf{K}_{m p}, \mathbf{K}_{s v}$ and $\mathbf{K}_{s p}$ are constant gain matrices. Also, let us choose the force reflection gains in (4) and (5) to be

$$
\begin{aligned}
& \mathbf{C}_{2}=\mathbf{C}_{m f} \\
& \mathbf{C}_{3}=\mathbf{C}_{s f}
\end{aligned}
$$

where $\mathbf{C}_{m f}$ and $\mathbf{C}_{s f}$ are constant force reflection gain matrices. We choose the other controllers in (4) and (5) according to (3) to satisfy the full transparency conditions:

$$
\begin{aligned}
& \mathbf{C}_{1}=s^{2} \mathbf{I}+\mathbf{K}_{s v} s+\mathbf{K}_{s p} \\
& \mathbf{C}_{4}=-\left(s^{2} \mathbf{I}+\mathbf{K}_{m v} s+\mathbf{K}_{m p}\right) \\
& \mathbf{C}_{5}=\mathbf{C}_{s f}-\mathbf{I} \\
& \mathbf{C}_{6}=\mathbf{C}_{m f}-\mathbf{I}
\end{aligned}
$$

Remark 2. In order to implement $\mathbf{C}_{1}$ and $\mathbf{C}_{4}$ in (10), we need to measure or compute the acceleration of the master and the slave robots. We can omit the acceleration terms if good low-frequency transparency is enough in the desired application. However, requiring good transparency over both low and high frequencies justifies using accelerometers [2]. $\diamond$

Using (8), (9) and (10) in the master and the slave closedloop dynamics (6) and (7) result in

$$
\begin{aligned}
\Delta \ddot{\mathbf{x}} & =-\mathbf{K}_{m v} \Delta \dot{\mathbf{x}}-\mathbf{K}_{m p} \Delta \mathbf{x}+\mathbf{C}_{m f} \Delta \mathbf{f}+\mathbf{M}_{x m}^{-1}\left(\mathbf{q}_{m}\right) \Delta \mathbf{d}_{m} \\
\Delta \ddot{\mathbf{x}} & =\mathbf{K}_{s v} \Delta \dot{\mathbf{x}}+\mathbf{K}_{s p} \Delta \mathbf{x}-\mathbf{C}_{s f} \Delta \mathbf{f}-\mathbf{M}_{x s}^{-1}\left(\mathbf{q}_{s}\right) \Delta \mathbf{d}_{s}
\end{aligned}
$$

where $\Delta \mathbf{x}=\mathbf{x}_{m}-\mathbf{x}_{s}$ is the position tracking error and $\Delta \mathbf{f}=$ $\mathbf{f}_{h}-\mathbf{f}_{e}$ is the force tracking error. Assume that matrices $\mathbf{C}_{m f}$, $\mathbf{C}_{s f}$ and $\mathbf{C}_{m f}^{-1}+\mathbf{C}_{s f}^{-1}$ are invertible. Multiplying (11) by $\mathbf{C}_{m f}^{-1}$ and (12) by $\mathbf{C}_{s f}^{-1}$ and adding them together, we can find the dynamic equation governing the position tracking error

$$
\Delta \ddot{\mathbf{x}}+\mathbf{K}_{v} \Delta \dot{\mathbf{x}}+\mathbf{K}_{p} \Delta \mathbf{x}=\boldsymbol{\Psi}_{x m}\left(\mathbf{q}_{m}\right) \Delta \mathbf{d}_{m}-\boldsymbol{\Psi}_{x s}\left(\mathbf{q}_{s}\right) \Delta \mathbf{d}_{s}
$$

where

$$
\begin{aligned}
\mathbf{K}_{v} & =\left(\mathbf{C}_{m f}^{-1}+\mathbf{C}_{s f}^{-1}\right)^{-1}\left(\mathbf{C}_{m f}^{-1} \mathbf{K}_{m v}+\mathbf{C}_{s f}^{-1} \mathbf{K}_{s v}\right) \\
\mathbf{K}_{p} & =\left(\mathbf{C}_{m f}^{-1}+\mathbf{C}_{s f}^{-1}\right)^{-1}\left(\mathbf{C}_{m f}^{-1} \mathbf{K}_{m p}+\mathbf{C}_{s f}^{-1} \mathbf{K}_{s p}\right) \\
\boldsymbol{\Psi}_{x m}\left(\mathbf{q}_{m}\right) & =\left(\mathbf{C}_{m f}^{-1}+\mathbf{C}_{s f}^{-1}\right)^{-1} \mathbf{C}_{m f}^{-1} \mathbf{M}_{x m}^{-1}\left(\mathbf{q}_{m}\right) \\
\boldsymbol{\Psi}_{x s}\left(\mathbf{q}_{s}\right) & =\left(\mathbf{C}_{m f}^{-1}+\mathbf{C}_{s f}^{-1}\right)^{-1} \mathbf{C}_{s f}^{-1} \mathbf{M}_{x s}^{-1}\left(\mathbf{q}_{s}\right)
\end{aligned}
$$

\section{Proposed disturbance observers}

In this section, we will design the disturbance observers in a way that full transparency and disturbance tracking are acheived under the control laws (4) and (5) with choices in (8), (9) and (10). Our proposed disturbance observers designed in the Cartesian space given the master and slave dynamics (1)-(2) are

$$
\begin{aligned}
\dot{\hat{\mathbf{d}}}_{m}= & -\mathbf{L}_{m} \hat{\mathbf{d}}_{m}+\mathbf{L}_{m}\left[\mathbf{M}_{x m}\left(\mathbf{q}_{m}\right) \ddot{\mathbf{x}}_{m}+\mathbf{N}_{x m}\left(\mathbf{q}_{m}, \dot{\mathbf{q}}_{m}\right)\right. \\
& \left.-\mathbf{f}_{h}-\mathbf{f}_{m}\right]+\mathbf{\Psi}_{x m}^{T}\left(\mathbf{q}_{m}\right)(\Delta \dot{\mathbf{x}}+\gamma \Delta \mathbf{x}) \\
\dot{\hat{\mathbf{d}}}_{s}= & -\mathbf{L}_{s} \hat{\mathbf{d}}_{s}+\mathbf{L}_{s}\left[\mathbf{M}_{x s}\left(\mathbf{q}_{s}\right) \ddot{\mathbf{x}}_{s}+\mathbf{N}_{x s}\left(\mathbf{q}_{s}, \dot{\mathbf{q}}_{s}\right)\right. \\
& \left.+\mathbf{f}_{e}-\mathbf{f}_{s}\right]+\mathbf{\Psi}_{x s}^{T}\left(\mathbf{q}_{s}\right)(-\Delta \dot{\mathbf{x}}-\gamma \Delta \mathbf{x})
\end{aligned}
$$

where $\gamma$ is an arbitrary positive constant. Also, $\mathbf{L}_{m}$ and $\mathbf{L}_{s}$ are constant gain matrices.

Note that in the 4-channel teleoperation control architecture, it is assumed that $\mathbf{f}_{h}$ and $\mathbf{f}_{e}$ are measured and the same measurements will be used in (18) and (19). The proposed nonlinear disturbance observers in (18) and (19) also need acceleration measurements. As mentioned before, the need for full transparency in a wide frequency range justifies using accelerometer - again, the same measurements will be needed in (18) and (19). Equations (1) and (2), along with (18) and (19), result in the following disturbance estimation error dynamics:

$$
\begin{aligned}
\dot{\hat{\mathbf{d}}}_{m} & =\mathbf{L}_{m} \Delta \mathbf{d}_{m}+\Psi_{x m}^{T}\left(\mathbf{q}_{m}\right)(\Delta \dot{\mathbf{x}}+\gamma \Delta \mathbf{x}) \\
\dot{\hat{\mathbf{d}}}_{s} & =\mathbf{L}_{s} \Delta \mathbf{d}_{s}+\Psi_{x s}^{T}\left(\mathbf{q}_{s}\right)(-\Delta \dot{\mathbf{x}}-\gamma \Delta \mathbf{x})
\end{aligned}
$$

Thoroughout the paper, we assume that the rate of change of the lumped disturbance is neglibgible in comparsion with the estimation error dynamics, i.e. $\dot{\mathbf{d}}_{m} \cong \mathbf{0}$ and $\dot{\mathbf{d}}_{s} \cong \mathbf{0}$. This assumption is not overly restrictive and is commonly encountered in the robotics literature (see, for example, [10]). Thus, the following disturbance estimation error dynamics result from (20) and (21) for the master and the slave, respectively:

$$
\begin{aligned}
\Delta \dot{\mathbf{d}}_{m} & =-\mathbf{L}_{m} \Delta \mathbf{d}_{m}-\boldsymbol{\Psi}_{x m}^{T}\left(\mathbf{q}_{m}\right)(\Delta \dot{\mathbf{x}}+\gamma \Delta \mathbf{x}) \\
\Delta \dot{\mathbf{d}}_{s} & =-\mathbf{L}_{s} \Delta \mathbf{d}_{s}-\boldsymbol{\Psi}_{x s}^{T}\left(\mathbf{q}_{s}\right)(-\Delta \dot{\mathbf{x}}-\gamma \Delta \mathbf{x})
\end{aligned}
$$

Remark 3. The terms $\boldsymbol{\Psi}_{x m}^{T}\left(\mathbf{q}_{m}\right)(\Delta \dot{\mathbf{x}}+\gamma \Delta \mathbf{x})$ and $\Psi_{x s}^{T}\left(\mathbf{q}_{s}\right)(\Delta \dot{\mathbf{x}}+\gamma \Delta \mathbf{x})$ in (18) and (19) are new and do not exist in the nonlinear disturbance observer proposed 
by [10]. These new terms will help us to guarantee global force tracking, global exponential position tracking and disturbance tracking in our teleoperation system. $\diamond$

The following two theorems state the main results of this paper.

Theorem 1: Consider the teleoperation system subject to disturbances described by (1) and (2). The master and the slave disturbance observers are given in (18) and (19). Then the disturbance observer based control laws given in (4) and (5) with choices in (8), (9) and (10), guarantee global asymptotic stability of the disturbance tracking error, the position tracking error, and the force tracking error if the following conditions hold:

1) $\mathbf{L}_{m}=\mathbf{L}_{m}^{T}>\mathbf{0}$ and $\mathbf{L}_{s}=\mathbf{L}_{s}^{T}>0$ are constant symmetric and positive definite matrices,

2) $\mathbf{K}_{v}$ given by (14) exists and is a constant symmetric and positive definite matrix satisfying $\mathbf{K}_{v}>\gamma \mathbf{I}$,

3) $\mathbf{K}_{p}$ given by (15) exists and is a constant symmetric and positive definite matrix,

4) $\dot{\mathbf{d}}_{m} \cong \mathbf{0}$ and $\dot{\mathbf{d}}_{s} \cong \mathbf{0}$, i.e., the rates of change of disturbances acting on the master and the slave robots are negligible in comparison with the estimation error dynamics (20) and (21).

Proof: Proof can be found in [12].

The previous theorem addresses the case when we have slow-varying disturbances. In tha case of fast-varying disturbances, the tracking errors will be globally uniformly ultimately bounded [12]. The next theorem states that the disturbance tracking and the position tracking errors of the teleoperation system can converge exponentially to zero under certain conditions.

Theorem 2: Consider the teleoperation system subject to disturbances described by (1) and (2). The master and the slave disturbance observers are given in (18) and (19). Under the control laws (4) and (5) with choices in (8), (9) and (10), the disturbance tracking and position tracking errors converge exponentially to zero provided that the conditions of Theorem 1 hold and $\gamma \leq \sqrt{\lambda_{\max }\left(\mathbf{K}_{p}+\gamma \mathbf{K}_{v}\right)}$ where $\lambda_{\max }($. represents the maximum eigenvalue of a matrix.

Proof: Proof can be found in [12].

Remark 4. As it is shown in Theorem 2, the minimum exponential rate of convergence for the disturbance tracking and the position tracking errors to the origin is equal to $\frac{\lambda_{\min }\left(\boldsymbol{\Gamma}_{2}\right)}{\lambda_{\max }\left(\boldsymbol{\Gamma}_{1}\right)}$. On the other hand, we have $\lambda_{\max }\left(\boldsymbol{\Gamma}_{1}\right)=$ $\kappa_{1}=\max \left\{1, \lambda_{\max }\left(\mathbf{K}_{p}+\gamma \mathbf{K}_{v}\right)\right\} \quad$ and $\lambda_{\min }\left(\Gamma_{2}\right)=\kappa_{2}=$ $\min \left\{\lambda_{\text {min }}\left(\mathbf{K}_{v}-\gamma \mathbf{I}\right), \gamma \lambda_{\text {min }}\left(\mathbf{K}_{p}\right), \lambda_{\text {min }}\left(\mathbf{L}_{m}\right), \lambda_{\text {min }}\left(\mathbf{L}_{s}\right)\right\}$. Thus, one can simply determine the minimum rate of convergence by computing $\frac{\kappa_{2}}{\kappa_{1}} \cdot \diamond$

\section{SIMULATION STUDY}

In this section, computer simulations will illustrate the effectiveness of the proposed control scheme. Both the master and the slave robots are considered to be planar two-link manipulators with revolute joints. The Cartesian dynamics are [14]

$$
\begin{gathered}
\mathbf{M}_{x}(\mathbf{q})=\left[\begin{array}{cc}
m_{2}+\frac{m_{1}}{s_{2}^{2}} & 0 \\
0 & m_{2}
\end{array}\right] \\
\mathbf{V}_{x}(\mathbf{q}, \dot{\mathbf{q}})=\left[\begin{array}{ll}
V_{x_{1}}(\mathbf{q}, \dot{\mathbf{q}}) & V_{x_{2}}(\mathbf{q}, \dot{\mathbf{q}})
\end{array}\right]^{T}
\end{gathered}
$$

$$
\mathbf{G}_{x}(\mathbf{q})=\left[\begin{array}{ll}
m_{1} g \frac{c_{1}}{s_{2}}+m_{2} g s_{12} & m_{2} g c_{12}
\end{array}\right]^{T}
$$

where

$$
\begin{aligned}
V_{x_{1}}= & -\left(m_{2} l_{1} c_{2}+m_{2} l_{2}\right) \dot{q}_{1}^{2}-m_{2} l_{2} \dot{q}_{2}^{2}- \\
& \left(2 m_{2} l_{2}+m_{2} l_{1} c_{2}+m_{1} l_{1} \frac{c_{2}}{s_{2}^{2}}\right) \dot{q}_{1} \dot{q}_{2} \\
V_{x_{2}=}= & m_{2} l_{1} s_{2} \dot{q}_{1}^{2}+l_{1} m_{2} s_{2} \dot{q}_{1} \dot{q}_{2}
\end{aligned}
$$

Also, the forward kinematics and the Jacobian matrix are

$$
\begin{gathered}
\mathbf{h}(\mathbf{q})=\left[\begin{array}{ll}
x_{1} & x_{2}
\end{array}\right]^{T}=\left[\begin{array}{cc}
l_{1} c_{1}+l_{2} c_{12} & l_{1} s_{1}+l_{2} s_{12}
\end{array}\right]^{T} \\
\mathbf{J}(\mathbf{q})=\left[\begin{array}{cc}
l_{1} s_{2} & 0 \\
l_{1} c_{2}+l_{2} & l_{2}
\end{array}\right]
\end{gathered}
$$

where $l_{1}$ and $l_{2}$ are the lengths of the links, and $m_{1}$ and $m_{2}$ are the point masses of the links. Also, we have $s_{1}=\sin \left(q_{1}\right)$, $s_{2}=\sin \left(q_{2}\right), c_{1}=\cos \left(q_{1}\right)$ and $c_{2}=\cos \left(q_{2}\right)$.

In this simulation study, the remote environment and the human operator's hand are modeled as linear impedances with mass, damping and stiffness terms. We take $\mathbf{Z}_{e}(s)=$ $\left(m_{e} s^{2}+b_{e} s+k_{e}\right) \mathbf{I}$ and $\mathbf{Z}_{h}(s)=\left(m_{h} s^{2}+b_{h} s+k_{h}\right) \mathbf{I}$, where $\mathbf{I}$ is the identity matrix. In the simulations, the human hand parameters are chosen as in [15]. The remote environment is chosen to be a relatively stiff and damped medium, modeled by dampers and springs. These lead us to $m_{h}=$ $11.6 \mathrm{~kg}, b_{h}=17 \mathrm{Nsm}^{-1}, k_{h}=243 \mathrm{Nm}^{-1}$ and $m_{e}=0.0 \mathrm{~kg}, b_{e}=$ $800 \mathrm{Nsm}^{-1}, k_{e}=1000 \mathrm{Nm}^{-1}$.

The friction torques acting on the joints of the robots are generated based on the model in [16]. For the $i-t h$ joint of the robot, $i=1,2$, we have the frictions modeled as

$$
\begin{aligned}
\tau_{i_{\text {friction }}=}= & F_{c i} \operatorname{sgn}\left(\dot{q}_{i}\right)\left[1-\exp \left(\frac{-\dot{q}_{i}^{2}}{v_{s i}^{2}}\right)\right] \\
& +F_{s i} \operatorname{sgn}\left(\dot{q}_{i}\right) \exp \left(\frac{-\dot{q}_{i}^{2}}{v_{s i}^{2}}\right)+F_{v i} \dot{q}_{i}
\end{aligned}
$$

where $F_{c i}, F_{s i}, F_{v i}$ are the Coulomb, static, and viscous friction coefficients, respectively. The parameter $v_{s i}$ is the Stribeck parameter. In the simulations, the friction coefficients and the Stribeck parameter for the master and the slave are chosen as follows [3]:

$$
F_{c i}=0.49, F_{s i}=3.5, F_{v i}=0.15, v_{s i}=0.189, i=1,2
$$

In the simulations, we take the actual dynamic parameter values of the master and the slave robots to be

$$
\begin{array}{r}
m_{1 m}=2.3 \mathrm{~kg}, m_{2 m}=2.3 \mathrm{~kg}, l_{1 m}=0.5 \mathrm{~m}, l_{2 m}=0.5 \mathrm{~m} \\
m_{1 s}=1.5 \mathrm{~kg}, m_{2 s}=1.5 \mathrm{~kg}, l_{1 s}=0.5 \mathrm{~m}, l_{2 s}=0.5 \mathrm{~m}
\end{array}
$$

Assuming a maximum of $\pm 25 \%$ uncertainty in these parameters, we take the approximate values of these master and the slave parameters to be randomly deviated from the actual values: 


$$
\begin{gathered}
\hat{m}_{1 m}=2.82 \mathrm{~kg}, \hat{m}_{2 m}=2.36 \mathrm{~kg}, \hat{l}_{1 m}=0.62 \mathrm{~m}, \hat{l}_{2 m}=0.56 \mathrm{~m} \\
\hat{m}_{1 s}=1.16 \mathrm{~kg}, \hat{m}_{2 s}=1.29 \mathrm{~kg}, \hat{l}_{1 s}=0.38 \mathrm{~m}, \hat{l}_{2 s}=0.52 \mathrm{~m}
\end{gathered}
$$

The controllers $\mathbf{C}_{m}, \mathbf{C}_{s}, \mathbf{C}_{1}, \ldots$, and $\mathbf{C}_{6}$ are chosen as in (8), (9) and (10). The controllers and disturbance observer gains and initial conditions are chosen as

$$
\begin{array}{ccc}
\gamma=1 & & \\
\mathbf{K}_{m v}=10 \mathbf{I} & , & \mathbf{K}_{s v}=10 \mathbf{I} \\
\mathbf{K}_{m p}=20 \mathbf{I} & , & \mathbf{K}_{s p}=20 \mathbf{I} \\
\mathbf{C}_{m f}=\mathbf{I} & , & \mathbf{C}_{s f}=\mathbf{I} \\
\mathbf{L}_{m}=20 \mathbf{I} & , & \mathbf{L}_{s}=20 \mathbf{I} \\
\hat{\mathbf{d}}_{0 m}=\mathbf{0} & , & \hat{\mathbf{d}}_{0 s}=\mathbf{0} \\
\mathbf{K}_{v}=5 \mathbf{I} & , & \mathbf{K}_{p}=10 \mathbf{I}
\end{array}
$$

The above gains satisfy the conditions in Theorem 1 and the Theorem 2. Note that the master and the slave controller and observer gains have been chosen to be equal- this choice is not necessary but is one that results in identical closed-loop master and slave dynamics (11) and (12) when $\Delta \mathbf{d}_{m}=\mathbf{0}$ and $\Delta \mathbf{d}_{s}=\mathbf{0}$.

To start the simulations, there is no need to apply exogenous forces to the master and the slave, i.e., we take $\mathbf{f}_{h}^{*}=\mathbf{0}$ and $\mathbf{f}_{e}^{*}=\mathbf{0}$. Instead, we choose different values for initial joint positions of the master and the slave while assuming that both robots are initially at rest. We take the initial joint position vectors to be

$$
\mathbf{q}_{0 m}=\left[30^{\circ}, 135^{\circ}\right]^{T}, \mathbf{q}_{0 s}=\left[0,90^{\circ}\right]^{T}
$$

Figures 3 and 4 show the position and force tracking response of the teleoperation system when no disturbance observer is used. Because of the friction forces and the dynamic uncertainties present in the master and the slave, the control law without using disturbance observers fails to achieve good position and force tracking. As can be seen in Figure 3 , from $t \approx 2^{s e c}$ we have $\Delta \dot{\mathbf{x}} \approx \mathbf{0}$ and we have got a constant offset in position tracking errors from $t \approx 2^{\text {sec }}$, when no disturbance observer is used.
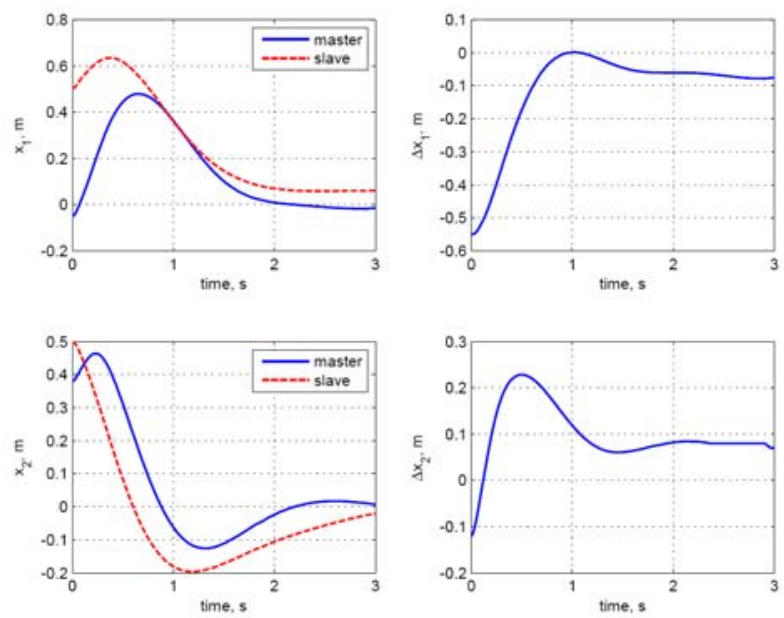

Fig. 3. Position tracking of the teleoperation system with conventional scheme (no disturbance observer).
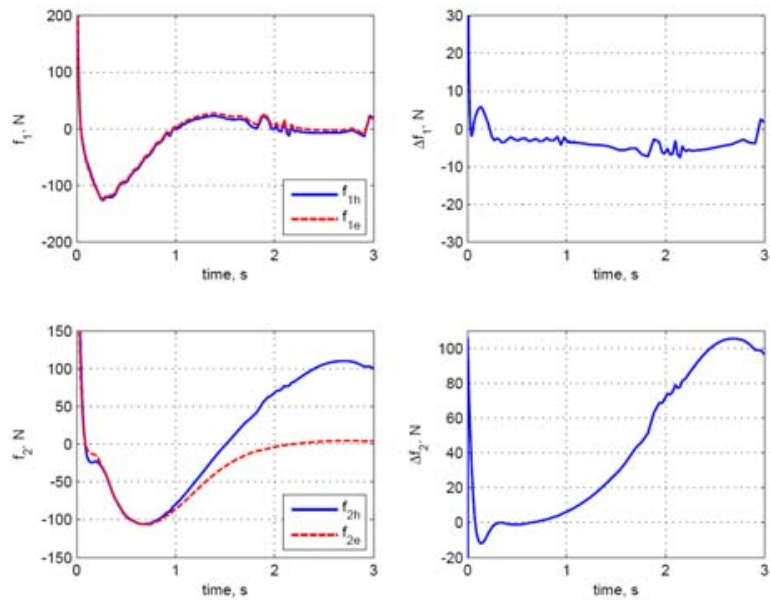

Fig. 4. Force tracking of the teleoperation system with conventional scheme (no disturbance observer).

Figures 5 and 6 show the distrubance tracking of the disturbance observer at the master and the slave sides, repectively. As it can be seen in Figure 3, the disturbance tracking error is small when there are no fast changes in the disturbances. In fact, the estimated disturbances tend towards the actual disturbances in the steady state.
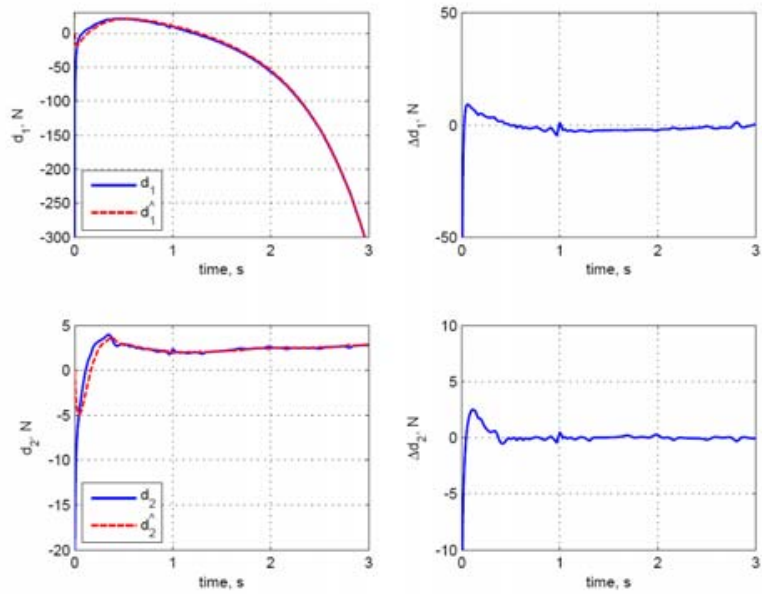

Fig. 5. Disturbance tracking at the master side.

Figures 7 and 8 show the position and force tracking response of the teleoperation system when disturbance observer is used. As it can be seen, good force and position tracking is achieved. Although we have disturbances with relatively fast dynamics our proposed control scheme achieves full transparency. We expect the minimum exponential convergence rate, as mentioned in Remark 5, to be equal to

$$
\begin{aligned}
& \frac{\kappa_{2}}{\kappa_{1}}= \\
& \frac{\min \left\{\lambda_{\min }\left(\mathbf{K}_{v}-\gamma \mathbf{I}\right), \gamma \lambda_{\min }\left(\mathbf{K}_{p}\right), \lambda_{\min }\left(\mathbf{L}_{m}\right), \lambda_{\min }\left(\mathbf{L}_{s}\right)\right\}}{\max \left\{1, \lambda_{\max }\left(\mathbf{K}_{p}+\gamma \mathbf{K}_{v}\right)\right\}} \\
& =\frac{4}{15}
\end{aligned}
$$

As it can be seen from the disturbance tracking and position 

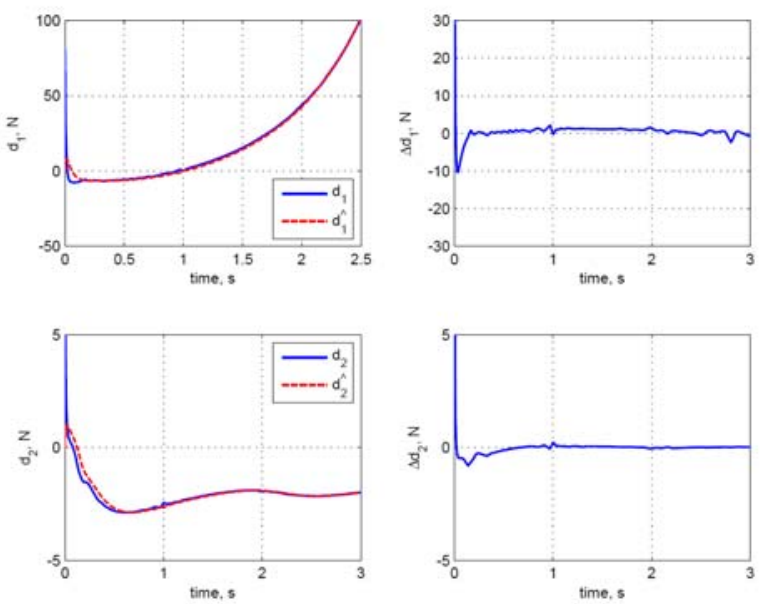

Fig. 6. Disturbance tracking at the slave side.

tracking plots, the slowest tracking speed belongs to $\Delta x_{2}$. In this case we have

$$
\ln \left(\frac{\Delta x_{2}\left(1^{\text {sec }}\right)}{\Delta x_{2}\left(0^{\text {sec }}\right)}\right) \approx \ln \left(\frac{0.14}{0.1}\right)=0.33 \geq \frac{\kappa_{2}}{\kappa_{1}}=\frac{4}{15}
$$
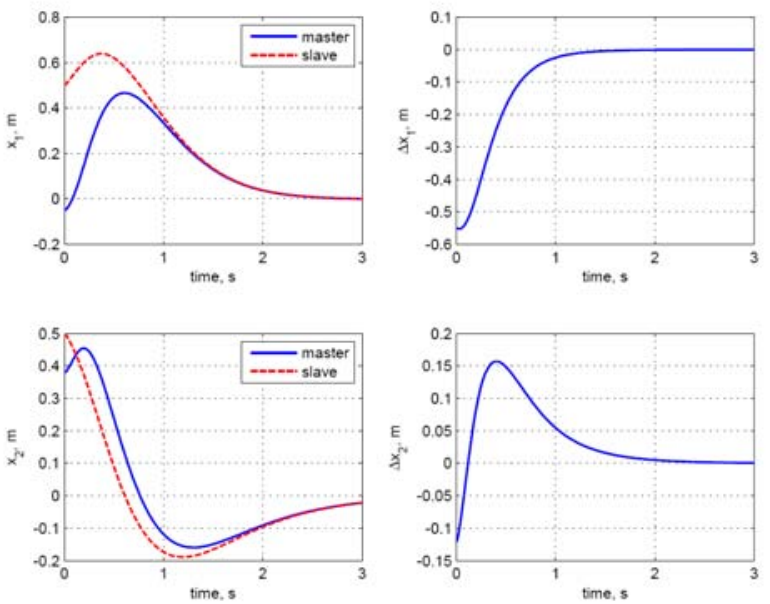

Fig. 7. Position tracking of the teleoperation system with disturbance observer-based controller.

\section{CONCLUSION}

In this paper, a novel control scheme has been proposed for nonlinear teleoperation systems subject to dynamic uncertainties and disturbances. The designed nonlinear disturbance observer-based controller is incorporated into the 4-channel bilateral teleoperation control framework. The proposed control scheme is able to suppress the disturbances present in the teleoperation system. Transparency, in terms of asymptotic convergence of the position and force tracking errors to zero and exponential convergence of disturbance tracking and position tracking errors to zero are achieved. The minimum exponential convergence rate can be adjusted to a desired value by proposed controller parameters. Simulations are done to show the effectiveness of the proposed approach.
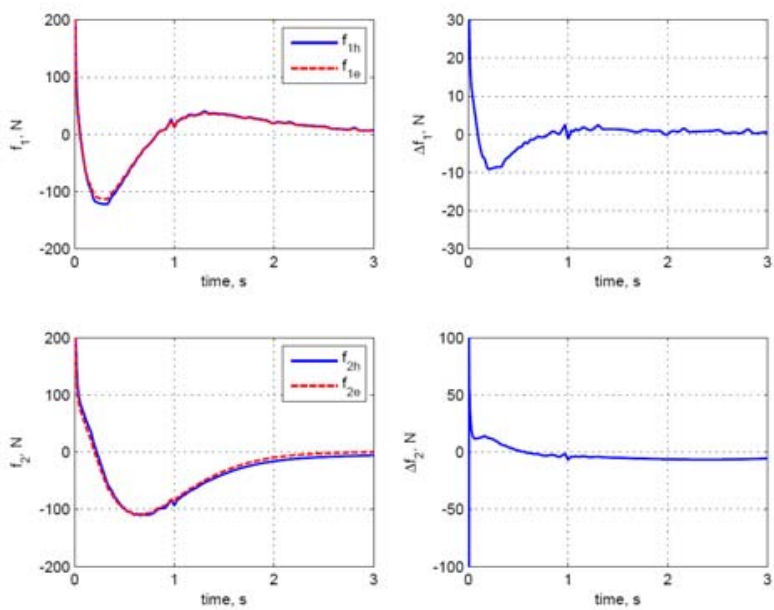

Fig. 8. Force tracking of the teleoperation system with disturbance observer-based controller.

\section{REFERENCES}

[1] K. Hashtrudi-Zaad and S. E. Salcudean, "Transparency in time delay systems and the effect of local force feedback for transparent teleoperation," IEEE Trans. Robot. Autom., vol. 18, no. 1, pp. 108$114,2002$.

[2] D. A. Lawrence, "Stability and transparency in bilateral teleoperation," IEEE Trans. Robot. Autom., vol. 9, pp. 624-637, Oct. 1993.

[3] N.V.Q. Hung, H.D. Tuan, T. Narikiyo, and P. Apkarian, "Adaptive control for nonlinearly parameterized uncertainties in robot manipulators," IEEE Trans. Cont. Syst. Technol., vol. 16, no. 3, pp. 458-468, 2008.

[4] K. Ohnishi, M. Shibata, and T. Murakami, "Motion control for advanced mechatronics," IEEE/ASME Trans. Mechatron., vol. 1, no. 1, pp. 56-67, Mar. 1996.

[5] K. S. Eom, I. H. Suh, and W. K. Chung, "Disturbance observer based path tracking control of robot manipulator considering torque saturation," in Proc. Int. Conf. Advanced Robot., 1997, pp. 651-657.

[6] S. Sankaranarayanan and F. Khorrami, "Model independent friction compensation," in Proc. American Control Conference, 1998, pp. 463 467.

[7] G. G. Zhang and J. Furusho, "Control of robot arms using joint torque sensors," in Proc. IEEE Int. Conf. Robot. Autom., 1997, pp. 3148 3153.

[8] S. Kim, J. Y. Lee, and D. K. Sung, "A shifted gamma distribution model for long-range dependent internet traffic," IEEE Commun. Lett. vol. 7, no. 3, pp. 124-126, Mar. 2003.

[9] S. Komada, N. Machii, and T. Hori, "Control of redundant manipulators considering order of disturbance observer," IEEE Trans. Ind. Electron., vol. 47, no. 2, pp. 413-420, Apr. 2000.

[10] W. H. Chen, D. J. Ballance, P. J. Gawthrop, and J. O'Reilly, "A nonlinear disturbance observer for robotic manipulators," IEEE Trans. Ind. Electron., vol. 47, no. 4, pp. 932-938, Aug. 2000.

[11] Y. Matsumoto, S. Katsura, and K. Ohnishi, "An analysis and design of bilateral control based on disturbance observer," in Proc. IEEE Int. Conf. Ind. Technol., 2003, pp. 802-807.

[12] A. Mohammadi, M. Tavakoli, and H. J. Marquez, "Disturbance observer based control of nonlinear haptic teleoperation systems," IET Control Theory Appl., 2011, In Press.

[13] M. Tavakoli, A. Aziminejad, R.V. Patel, and M. Moallem, "Highfidelity bilateral teleoperation systems and the effect of multimodal haptics," IEEE Trans. Syst., Man Cybern. B, bern., vol. 37, no. 6, pp. 1512-1528, Dec. 2007.

[14] J. J. Craig, Introduction to Robotics: Mechanics and Control, Upper Saddle River, NJ: Pearson Prentice Hall, 2005.

[15] K. Kosuge, Y. Fujisawa, and T. Fukuda, "Control of mechanical system with man-machine interaction," in Proc. IEEE/RSJ Int. Conf. Intell. Robots Syst., 1992, pp. 87-92.

[16] B. Armstrong-Hélouvry, P. Dupont, and C. Canudas de Wit, "A survey of models, analysis tools and compensation methods for the control of machines with friction," Automatica, vol. 30, no. 7, pp. 1083-1138, 1994. 